\title{
Model for End-stage Liver Disease
}

\author{
Ashwani K. Singal, Patrick S. Kamath \\ Division of Gastroenterology and Hepatology, Mayo Clinic, Rochester, MN, USA
}

\begin{abstract}
Model for end-stage liver disease (MELD) score, initially developed to predict survival following transjugular intrahepatic portosystemic shunt was subsequently found to be accurate predictor of mortality amongst patents with end-stage liver disease. Since 2002, MELD score using 3 objective variables (serum bilirubin, serum creatinine, and institutional normalized ratio) has been used worldwide for listing and transplanting patients with end-stage liver disease allowing transplanting sicker patients first irrespective of the wait time on the list. MELD score has also been shown to be accurate predictor of survival amongst patients with alcoholic hepatitis, following variceal hemorrhage, infections in cirrhosis, after surgery in patients with cirrhosis including liver resection, trauma, and hepatorenal syndrome (HRS). Although, MELD score is closest to the ideal score, there are some limitations including its inaccuracy in predicting survival in $\mathbf{1 5 - 2 0 \%}$ cases. Over the last decade, many efforts have been made to further improve and refine MELD score. Until, a better score is developed, liver allocation would continue based on the currently used MELD score. ( J CLIN EXP HePATOL 2013;3:50-60)
\end{abstract}

A llocation of organs for liver transplantation in the United States in the 1980s and early 1990s was prioritized based on the level of care required by the patient: hospitalized patients in the intensive care unit, hospitalized patients on the regular floor, and outpatient care. This approach had the potential of 'gaming' the system by keeping the patients in ICU in order to be transplanted. In 1996, a consensus conference mandating need for minimal criteria for listing the patients for liver transplantation (LT) introduced the Child-Pugh-Turcotte (CTP) score for liver allocation. ${ }^{1}$ CTP score is based on severity of 3 objective (serum albumin, serum bilirubin, and prothrombin time) and 2 subjective (ascites and encephalopathy) parameters. Subjective parameters vary with use of diuretics or paracenteses for ascites and use of lactulose for encephalopathy. CTP score introduced to some extent the concept of 'sicker patient first' with the introduction of status $1 \mathrm{~A}$ for patients with fulminant hepatic failure,

Keywords: MELD, Liver transplantation, Cirrhosis

Received: 2.11.2012; Accepted: 20.11.2012; Available online: 1.12 .2012

Address for correspondence: Patrick S. Kamath, Division of Gastroenterology and Hepatology, Mayo Clinic, Rochester, MN, USA.

E-mail: kamath.patrick@mayo.edu

Abbreviations: MELD: model for end-stage liver disease; LT: liver transplantation; CTP: Child-Pugh-Turcotte; ESLD: end-stage liver disease; UNOS: United Network for Organ Sharing; TIPS: transjugular intrahepatic portosystemic; GFR: glomerular filtration rate; MDRD: modification of diet in renal disease; FHF: fulminant hepatic failure; QALY: quality adjusted life years; MLP: multi-layer perceptron; SOFA: sequential organ failure assessment; SOFT: survival outcomes following transplantation; BAR: balance risk; EDC: extended donor criteria; UKELD: UK end stage liver disease score; deMELD: drop-out equivalent MELD; SLK: simultaneous liver kidney transplantation; AH: alcoholic hepatitis; DFI: discriminate function index; HVPG: hepatic venous pressure gradient; VH: variceal hemorrhage

http://dx.doi.org/10.1016/j.jceh.2012.11.002 primary non-function or hepatic artery thrombosis within 7 days of transplantation, and decompensated Wilson's disease. Organ allocation for patients with end-stage liver disease (ESLD), however, largely depended on waiting time on the list. In 1998, the Institute of Medicine mandated that patients be allocated organs based on their disease severity and risk of death rather than on waiting time. ${ }^{2}$

\section{DEVELOPMENT OF THE MODEL FOR END-STAGE LIVER DISEASE SCORE AND ADOPTION BY THE UNITED NETWORK FOR ORGAN SHARING FOR LIVER ALLOCATION}

February 27, 2002 was a historical day when the MELD score was adopted and approved by the United Network for Organ Sharing (UNOS) as a score to allocate organs for patients awaiting liver transplantation (LT) in the United States. ${ }^{3}$ This score changed the policy of organ allocation not only upholding the concept of "patient comes first" but also "sickest patient comes first". That is, the patient most at risk for mortality would be at the highest priority for organ allocation.

MELD score was developed by a group of researchers at the Mayo Clinic initially as a model to predict survival following transjugular intrahepatic portosystemic (TIPS) for refractory variceal bleeding or refractory ascites. ${ }^{4}$ The model was later shown to quite accurately predict 3 months mortality amongst patients with chronic end-stage liver disease awaiting LT. ${ }^{5,6}$ As the score was objective and could predict mortality at 3 months with higher accuracy than the CTP score, allocation of livers for transplantation became MELD based, deemphasizing the concept of waiting time. ${ }^{3,7}$

The score was initially named as Mayo model for endstage liver disease (MELD) score reflecting the institution 
where the score was developed. With the acceptance of this score by the UNOS for organ allocation, the model was renamed as model for end-stage liver disease. This allowed wider acceptability of the score keeping the same abbreviation of MELD. ${ }^{7,8}$ Other changes made to the score by the UNOS were: capping serum creatinine at $4 \mathrm{mg} / \mathrm{dl}$, capping the score at 40 , and setting the lower limit for each component of the score to 1 in order to avoid negative scores. Further, etiology of the liver disease as a factor was removed from the model, as this did not impact mortality amongst patients with end-stage liver disease awaiting $\mathrm{LT}^{5}$

\section{COMPARISON OF CHILD-PUGH-TURCOTTE VERSUS MODEL FOR END-STAGE LIVER DISEASE SCORE}

Mortality and MELD score are linearly correlated amongst patients with end-stage liver disease listed for LT with 3 month mortality estimated to be $4 \%, 27 \%, 76 \%, 83 \%$, and $100 \%$ for MELD scores of $<10,10-19,20-29,30-39$, and 40 or more respectively. Predictive ability of any model or score is given by c-statistic, which ranges between 0 and 1. A ' $c$ ' statistic of 0.7 is considered clinically useful and a c-statistic of 0.8 or more qualifies for an accurate model. This means that if 2 patients are on the waiting list, a model with a c-statistic of 0.8 would be $80 \%$ accurate in predicting death of the patient with the higher score earlier than the patient with the lower score. In the initially developed model, c-statistic for MELD score was 0.87 , which was superior to CTP score with c-statistic of 0.84 . Other studies including meta-analyses have shown that both CTP and MELD scores are predictive of waitlist mortality. ${ }^{9,10}$ However, the MELD score allows finer stratification than CTP score. Further, MELD incorporates serum creatinine, a factor which is important in predicting survival in patients with liver disease. ${ }^{11-13}$

\section{COMPONENTS OF THE MODEL FOR END-STAGE LIVER DISEASE SCORE AND LIMITATIONS OF EACH COMPONENT}

MELD score is calculated using serum bilirubin, serum creatinine, and International Normalized Ratio (INR) and is given by the formula $9.57 \times$ loge (creatinine) $+3.78 \times$ loge (total bilirubin) $+11.2 \times$ loge $($ INR $)+6.43$. The score can be calculated using online website www.mayoclinicorg./gi-rst/ mayomodel5 html.

\section{Serum Bilirubin}

Problems with using serum bilirubin as a variable are the potential for error in measurement and elevation in the presence of renal failure. ${ }^{14}$ Further, total bilirubin which is used for calculation of the MELD score may change due to increased indirect bilirubin from hemolysis, blood transfusion, and genetic variability of the bilirubin metabolism. The predictive ability of the MELD score does not change whether direct or total bilirubin is used, and hence the total bilirubin is used for calculating the MELD score in clinical practice. ${ }^{15}$

\section{Serum Creatinine}

For the purposes of organ allocation, serum creatinine is set at a lower limit of $1 \mathrm{mg} / \mathrm{dl}$ with a ceiling at $4 \mathrm{mg} / \mathrm{dl}$. Any patient who received dialysis $\geq 2$ times in the previous week irrespective of the serum creatinine value is determined to have a serum creatinine of $4 \mathrm{mg} / \mathrm{dl}$. This ceiling value for serum creatinine is arbitrary and in one study, increasing the upper limit of serum creatinine to 5.5 had a small impact of $2.5 \%$ in 3 months mortality amongst wait listed candidates but improved accuracy of the MELD score in predicting 3 months mortality. ${ }^{16}$ The limitation of inclusion of serum creatinine is variation in its value depending on the method of measurement: colorimetric or enzymatic. Further, in the presence of high serum bilirubin, the colorimetric method is unreliable and underestimates the value of creatinine; the enzymatic method is preferred for measuring the serum creatinine in such situations. ${ }^{17}$ Another limitation is gender with lower value amongst females compared to men for the same level of renal function. ${ }^{18}$ A study comparing glomerular filtration rate (GFR) based on Modification of Diet in Renal Disease (MDRD) formula and serum creatinine showed similar results in estimating waitlist mortality. ${ }^{19}$ Another study using corrected MELD with estimated GFR was unable to predict short-term mortality at 3-6 months but was better in predicting mortality at $9-12$ months after listing. ${ }^{20}$ In contrast, true GFR estimated using iohexol clearance is a better predictor of the renal function compared to MDRD or serum creatinine estimation as the latter methods tend to overestimate true GFR. ${ }^{21}$

\section{INR}

The INR has a sigmoid shape effect on the 3 months mortality of wait listed candidates with maximum effect between INR of 1 and 3. INR measured using thromboplastin obtained from patients on anticoagulant therapy leads to variations in the inter-laboratory readings on the INR values. ${ }^{22,23}$ Combined with geographic variation on the MELD threshold for transplantation, this limitation significantly impacts odds of liver allocation. ${ }^{22}$ Further, INR is prone to be confounded by use of warfarin. Use of liver specific thromboplastin using plasma from patients with cirrhosis instead of plasma from patients on warfarin eliminates this discrepancy and variation across laboratories. ${ }^{24}$ However, this method is expensive and adds to confusion in ordering the same tests for different indications. In one study amongst patients with cirrhosis on stable 
anticoagulation, model without INR was less accurate than the original MELD model suggesting that even in anticoagulated patients MELD model should be used for estimation of prognosis. ${ }^{25}$

\section{Other Variables}

Etiology of liver disease was initially included into the model but was later shown to be not predictive of outcomes and was then removed from the model. ${ }^{5}$ Similarly, complications of cirrhosis and portal hypertension such as ascites, variceal bleeding, or hepatic encephalopathy being components of CTP score did not significantly add to accuracy of MELD score suggesting that these complications usually reflect the status of underlying liver function. $^{26}$

\section{IMPACT OF IMPLEMENTING MODEL FOR END-STAGE LIVER DISEASE SCORE}

\section{Impact on Outcomes}

Introduction of MELD score for organ allocation in the United States in the very first year resulted in about $12 \%$ reduction in waitlist mortality. ${ }^{7}$ This trend continued in later years with reduction in total number of deaths on waitlist from 2046 in 2001 to 1364 in 2005 with reduction in waiting time from 656 days to 416 days. ${ }^{27}$ Part of this reduction was due to increase in number of donor livers from 4671 in 2001 to 5160 in 2005 . However, in spite of accounting for this, policy of allocating livers based on MELD score was responsible for this reduction as similar reduction in waitlist mortality did not occur amongst patients with fulminant hepatic failure (FHF). ${ }^{28}$

Studies have shown association of pre-LT MELD score with the hospital resource utilization such as operative time, use of red blood cell transfusions, duration of stay in the intensive care unit and total hospital stay and charges. In one study, MELD score of more than 23 predicted a higher morbidity and prolonged ICU stay. ${ }^{29}$ In another study, there was about $55 \%$ increased cost of transplanting a patient as compared to pre-MELD era. ${ }^{30}$ However, data on increased resource utilization since the implementation of MELD score are controversial with no such change reported in a large database retrospective study. ${ }^{31}$ However, there is also significant improvement in quality of life resulting in dynamic improvement in the cost to quality adjusted life years (QALY) ratio especially after 3-5 years of follow up amongst patients with high MELD scores prior to transplantation. ${ }^{32}$

\section{Impact on Disparities in Liver Transplantation}

Ethnic disparities on waitlist mortality and receipt of liver transplant within 3 years of registering have reduced. ${ }^{33,34}$ Incorporation of serum creatinine into the model resulted in gender disparities in receipt of transplant and higher waitlist mortality among women by $13 \%$ compared to men. ${ }^{35}$ This is due to the fact that for the given renal function, women tend to have lower serum creatinine compared to men due to lower muscle mass in women.

\section{Model for End-stage Liver Disease and Post- transplant Survival}

In spite of transplanting patients with a higher MELD score, post-LT survival did not change in the MELD era. Post-transplant survival is a multidimensional non-linear issue and depends upon multiple recipient and donor factors along with experience of transplant center. In one study, use of multi-layer perceptron (MLP) using 18 different recipient and donor variables was better predictor of post-transplant outcomes as compared to MELD and sequential organ failure assessment (SOFA) scores. ${ }^{36}$ In another study, 3 months post-transplant mortality was predicted by a SOFT (survival outcomes following transplantation) score incorporating 18 recipient and donor factors in addition to MELD score. ${ }^{37}$

In order to match graft with the MELD score and other recipient factors, a balance risk (BAR) score has been suggested in order to achieve a balance between waitlist mortality and post-transplant outcomes. ${ }^{38}$ In one study, combination of 3 extended donor criteria (EDC): age, steatosis $>30 \%$ and cold ischemia time with MELD > 28 predicted graft failure. ${ }^{39}$ Worsening MELD score or delta-MELD (current MELD-maximum score in the last 3 months) has been shown to impact post-transplant outcome, ${ }^{40}$ and one should avoid graft with $>1$ EDC for such patients. ${ }^{41}$ Similar observations by another study on patients with Hepatitis B virus (HBV) related liver disease and MELD > 29 showed that downgrading MELD score using anti-HBV drugs improved outcomes of LT compared to emergency LT. ${ }^{42}$ In this respect, product of age and delta-MELD less than 1600 may be required for optimal post-transplant outcomes. ${ }^{43}$

\section{Impact on Liver Allocation}

The aims of liver allocation are to reduce wait-list mortality and achieve significant transplant benefit. Although, sickest patients are expected to derive most transplant benefit, disease severity also impacts immediate post-transplant outcomes. Hence, in clinical practice, a balanced approach is needed to optimize liver allocation. ${ }^{44}$ Etiology of liver disease is not factored into the calculation of MELD score; patients with viral etiology of cirrhosis and MELD > 15 had significantly lower survival than alcoholic cirrhosis patients with similar MELD suggesting that viral cirrhosis patients may be disadvantaged in the MELD allocation policy. ${ }^{45}$ For a given MELD score between 15-17 and 24-40, a patient with higher serum creatinine is shown to have higher waitlist mortality compared to a patient with lower serum creatinine. This factor, if taken into 
consideration, would affect the liver allocation. ${ }^{12}$ Laboratory variations of INR and serum creatinine across transplant centers also result in variations in the MELD score. This combined with geographic variation results in a varying MELD threshold for receiving organs. ${ }^{46}$ In addition, allocation of a specific score for patients with Hepatocellular carcinoma (HCC) and other conditions ${ }^{47}$ significantly impacts odds of liver allocation. ${ }^{22}$ In this respect, normalization of MELD score based on corrected value of each variable normalized to the maximal normal value $\left(V_{\max }\right)$ of each laboratory and given as: corrected value $=$ measured value $\times V_{\max }$ of lab $1 / V_{\max }$ of lab 2 may optimize liver allocation. ${ }^{48}$

\section{Model for End-stage Liver Disease Exception Points and Liver Allocation}

Patients with HCC with lower MELD have a risk of progression of the tumor while waiting for transplant, leading to death or progression of disease which may exclude them from receiving an organ. On the other hand, HCC patients with higher biological MELD have been shown to have poor post-transplant survival compared to comparable MELD in non-HCC patients. ${ }^{49}$ Therefore, in February 2003, UNOS accepted a policy of awarding MELD exception points for HCC patients within Milan criteria. This change along with the documentation of post-LT outcomes for HCC within Milan criteria to be as good as for any other indication, has resulted in the proportion of transplants performed for HCC increasing from $4.6 \%$ in during $1997-2002$ to $26 \%$ during $2002-2007 .{ }^{49}$ Therefore, in March 2005, this policy was modified to award 22 MELD exception points instead of 24 points to patients with HCC to more accurately reflect their risk of dying. ${ }^{50}$ Even with this policy, the odds of a patient with HCC receiving the organ remains substantially higher compared to non-HCC patient. ${ }^{51,52}$ On the other hand, about $29 \%$ of patients with HCC are dropped from the waiting list due to tumor progression suggesting consideration of other factors such as number of tumors, alphafetoprotein levels, tumor biology, and biological or calculated MELD score for transplanting HCC patients. ${ }^{49,53}$ However, many of these factors are also associated with tumor recurrence after transplantation and a higher probability of drop out risk from the waiting list is directly correlated with a higher risk of recurrence of HCC after transplantation. ${ }^{54}$ Hence a balanced approach is needed to optimize use of livers for transplanting HCC patients aiming at maintaining post-transplant survival as well as optimizing the chance of receiving a transplant. In this regard, the concept of drop-out equivalent MELD (deMELD) points has been introduced taking into consideration the risk of drop out from the waiting list based on MELD score and other HCC characteristics. For example, two patients with similar MELD scores would be given different exception points based on their drop out risk from the waiting list after considering factors such as number of tumors, maximum tumor size, age of the patient, etiology of liver disease, and AFP levels. ${ }^{55}$

\section{Model for End-stage Liver Disease and Simultaneous Kidney Transplantation}

Due to incorporation of serum creatinine into the model, there has been an increase in the proportion of simultaneous liver kidney transplantation (SLK) from 2 to $3 \%$ during 1994-2001 to 4-7\% during 2002-2010 and increased incidence of post-transplant end-stage renal disease. ${ }^{56,57}$ However, post-transplant outcomes have not worsened in the MELD era. ${ }^{57,58}$ Increasing use of SLK has raised concern about the shortage of donor kidneys. Guidelines on allocating simultaneous kidney to LT recipients as laid down by a consensus group are not perfect. ${ }^{59}$ In this respect, there is an unmet need for biomarkers to accurately predict reversibility of the renal function after LT.

\section{Low Model for End-stage Liver Disease Score and Liver Transplantation}

Patients with MELD score of less than 15 who receive a transplant do worse than patients with a similar score who do not receive a transplant with 3 month post-LT mortality being about 3.6 times higher with LT for MELD 6-11, and 2.4 times higher for MELD 12-14. ${ }^{60}$ However, this may not be true for living donor liver transplantation for non-HCC patients as they still derived a significant survival benefit compared to patients waiting for deceased donor due to reduced wait time and better graft quality. ${ }^{61}$ This was confirmed in a study analyzing the UNOS database where transplant benefit of patients with MELD < 16 depended upon the quality of graft they received, and poor survival benefit amongst these patients was due to receipt of grafts with the highest donor risk index. ${ }^{62}$ Transplants for patients with low MELD score using high risk or marginal grafts are also associated with increased length of stay and cost of transplantation. ${ }^{63}$

\section{APPLICATIONS OTHER THAN LIVER TRANSPLANTATION FOR CIRRHOSIS}

\section{Referral for Hospice Care}

Hospice care may be useful adjunct to coordinate care of patients awaiting liver transplantation. In addition, patients who are not candidates for liver transplantation and are likely to die within 6 months may be referred for hospice care. ${ }^{64}$ MELD can accurately guide treating physicians on making such decisions. In one study, median MELD at the time of admission to hospice care program was 21 with median length of stay being 38 days. There was a linear correlation of length of stay and MELD score. ${ }^{65}$ In another study, MELD $>24$ could accurately predict mortality at 30 days in about $80 \%$ cases. 


\section{Fulminant Hepatic Failure}

Data on whether MELD score can predict mortality in FHF are controversial. In one study, MELD score was accurate in predicting the 30 day mortality amongst patients receiving LT for non-acetaminophen related FHF. ${ }^{66}$ Similarly, another study reported on adults with non-acetaminophen FHF showed MELD as good as King's college criteria. ${ }^{67}$ However, in a US multicenter study on patients with hepatitis A induced FHF, MELD was unable to predict outcome of patients on the LT list with a cstatistic of only $0.7{ }^{68}$ However, there were only 4 deaths and therefore the significance of the study is unclear. The most common cause of mortality amongst FHF patients is cerebral edema and it is likely that other factors including intra cranial pressure are important in predicting waitlist mortality of FHF patients. In a prospective study from Denmark, MELD was an important variable in predicting onset of FHF amongst patients with acetaminophen overdose. ${ }^{69}$ However, after the onset of FHF, MELD score was no more important in predicting mortality. Whether patients with FHF should be considered status $1 \mathrm{~A}$ and be prioritized over end-stage liver disease patients irrespective of MELD scores was addressed in a study using Scientific Registry of Transplant Recipients database. The findings showed that patients with endstage liver disease and MELD score $>40$ had higher waitlist mortality compared to status $1 \mathrm{~A}$ patients suggesting that these patients be prioritized over status $1 \mathrm{~A}$ patients in allocation of livers. However, further studies are needed to confirm these findings before implementing any change in policy. ${ }^{70}$

\section{Alcoholic Hepatitis}

MELD score accurately predicts outcome in patients with alcoholic hepatitis (AH). Studies comparing other scores and MELD score have shown conflicting data. Six studies have compared MELD and discriminate function index (DFI) scores amongst AH patients. MELD was similar to DFI for predicting 30 day mortality in 3 studies (c-statistic of $0.82,0.73$, and 0.89 for MELD >11, 21, and 18 vs. 0.86, 0.69 , and 0.81 respectively for DFI $>32^{71-73}$ ); superior to DFI in 2 studies in predicting 30 day mortality ( 0.83 for MELD $>22$ vs. 0.74 for DFI $>41^{74}$ or occurrence of complications of liver disease in one study ${ }^{75}$ ); and inferior to DFI in one study. ${ }^{76}$ Three studies comparing MELD and CTP showed 2 scores to be similar for mortality during the hospital stay ${ }^{72}$ or at 3 and 6 months ${ }^{6}$ while in the third study, CTP but not MELD was predictor of 90 day mortality. ${ }^{76}$ Apart from other limitations of MELD score, specific issue pertaining to AH is variation in MELD score cut off to accurately predict mortality ranging from 11 to 22 in different studies. American Association for Study of Liver Diseases (AASLD) guidelines recommend MELD cut-off of 18 to initiate corticosteroid therapy for patients with $\mathrm{AH} .{ }^{77}$

\section{Cirrhosis with Infections}

MELD score and renal failure including type of hepatorenal syndrome (HRS) predict outcome of infected cirrhotics while CTP score was not predictive. ${ }^{78}$ It is likely that MELD score contributes at both the stages and in patients with higher MELD score there should be a high index for suspecting infection and initiation of antibiotics. For example in a retrospective analysis on 256 Albanian patients with cirrhosis, MELD score was a predictor for occurrence of SBP and mortality. ${ }^{79}$ In another study on 111 hospitalized cirrhotics MELD was a predictor for SBP, increasing risk by about $11 \%$ for every increase in MELD score with 9.7 times higher odds for developing SBP at a MELD cut-off of $15 .{ }^{80}$ Amongst patients with community acquired pneumonia (CAP) in cirrhotics, a new score (MELD-CAP) incorporating extent of pneumonia and septic shock at admission was a better predictor of severe disease and mortality compared to CAP in patients without cirrhosis (OR 1.33 [1.091.52] and 1.21 [1.03-1.42]). ${ }^{81}$ MELD incorporated with serum sodium (MELD-Na) also accurately predicted mortality in patients with spontaneous bacterial empyema. ${ }^{82}$

\section{Transjugular Intrahepatic Portosystemic Placement}

As mentioned earlier, MELD score was initially developed to predict mortality after TIPS placement to manage patients with variceal bleeding or ascites refractory to routine measures. ${ }^{4}$ MELD score in predicting mortality after TIPS placement was superior to Emory score in one study and slightly superior or similar to CTP score in another study. ${ }^{83,84}$ MELD score $<18$ is ideal for TIPS placement, and those with MELD scores19-24 are borderline for successful outcome. Patients with MELD >24 are not optimal candidates for TIPS placement; TIPS may be carried out in these patients if they are candidates for liver transplantation. ${ }^{85}$ These guidelines have also been found to be useful for placement of TIPS amongst posttransplant patients and it is recommended that TIPS be carried out only if the MELD score is $<15$ in these patients. ${ }^{86}$

\section{Surgery Apart from Liver Transplantation}

Patients with decompensated cirrhosis may require abdominal or extra-abdominal non-transplant surgery in especially in the last 2 years of their life. ${ }^{87}$ The effect of the surgical procedure, blood loss, hypoxemia due to ascites and/or hydrothorax, and anesthetic agents all make a diseased liver prone to further deterioration with a potential risk for precipitating liver failure. ${ }^{88}$ Severity of liver disease predicting outcome after surgery has been known for years. Earlier, the risk used to be gauged using the CTP stage with $10 \%, 30 \%$, and $82 \%$ postoperative mortality amongst patients with CTP stages A, B, and C respectively. ${ }^{89}$ Type of 
surgery (emergency vs. elective) has been associated with outcome. Amongst patients with CTP class A, B, and C mortality for emergency surgery is $22 \%, 38 \%$, and $100 \%$ respectively. ${ }^{90}$ Since the introduction of MELD score, retrospective studies have confirmed MELD score to be predictive of outcome following surgery other than LT. ${ }^{91,92}$ In the largest retrospective study addressing this issue reported from the Mayo Clinic analyzing 772 patients undergoing various kinds of surgeries, MELD score was an important variable in predicting outcome after surgery for short-term (7, 30, and 90 days) and long-term outcomes at 1 or 5 years. Other important predictors were ASA class and recipient age with addition of 5.5 MELD points for ASA class IV and 3 MELD points for age more than 70 years. ${ }^{92}$ Emergency surgery was not predictive when the model was also controlled for the MELD score in addition to other variables. Based on MELD score, age, and ASA status one can predict outcomes after surgery using an online model at $h t t p: / / w w w$.mayoclinic.org/meld/ mayomodel9.html. This model can help in counseling patients and physicians on the risk of surgery. The association of risk of death after surgery and the MELD score $>8$ was linear and it is generally believed that patients with MELD score $<10$ can tolerate surgery, those with 10-15 MELD score may be considered, and patients with MELD score $>15$ should avoid an elective surgery. This recommendation has been validated in other studies on different populations. ${ }^{93,94}$ However, a study from Korea including a large number of patients with hepatitis B related cirrhosis reported that the model tends to overestimate mortality at more than $\geq 1$ year after surgery ${ }^{95}$ as the long-term outcome may potentially be confounded by comorbidities and other factors.

\section{Comparing Model for End-stage Liver Disease and Child-Pugh-Turcotte for Outcome After Surgery}

Many studies have compared MELD with CTP stage in predicting mortality after surgery. In one study, 3 month mortality rates amongst 3 respective CTP stages were $2 \%, 22 \%$, and $55 \%$ and similar rates at MELD of 6-9, 10-14, 15-19, $20-24$, and $>24$ were $3.5 \%, 8.9 \%, 14.3 \%, 12.5 \%$, and $63.6 \%$ respectively. ${ }^{96}$ In yet another study, perioperative mortality rates based on CTP stage were $10 \%, 17 \%$, and $63 \%$ while similar rates at MELD of $\langle 10,10-15$, and $>15$ were $9 \%$, $19 \%$, and $54 \%$ respectively. ${ }^{97}$ Amongst both the studies, CTP stage emerged better predictor of perioperative mortality compared to MELD score. In contrast, integrated MELD score was superior to CTP stage for predicting postoperative mortality. ${ }^{94}$ In another study, CTP and MELD scores were similar in predicting outcome after elective surgery but only fairly after urgent surgery in cirrhotics. ${ }^{98}$

\section{Type of Surgery}

For the same MELD score, mortality is higher in some studies for intra-abdominal surgery compared to abdominal wall surgeries including surgery for umbilical hernia. Amongst 220 cirrhotics undergoing cholecystectomy, a common surgery in general population including cirrhotics, the procedure was safe with no perioperative mortality across MELD range 8-27. However, a higher postoperative complication rate was noted for patients with MELD > $13 .{ }^{99}$ MELD score remains a powerful predictor of outcomes for head and neck cancer surgery at a cutoff of 9.7 ( $23 \%$ vs. $3 \%$ postoperative mortality; $P=0.03) .{ }^{100}$

Regarding elective cardiac surgery, if possible, least invasive option of angioplasty with or without stent placement should be considered. One should also avoid coated stents, as they require need for anticoagulants and antiplatelet agents such as clopidogrel. Cardiac surgery is safe in CTP stage A, can be considered in select CTP-B stage patients and should be avoided in CTP-C stage. ${ }^{101,102}$ MELD score remains a predictor of outcome after tricuspid valve surgery ${ }^{103}$ and left ventricular assist device placement. ${ }^{104,105}$

\section{HCC Resection in Cirrhosis}

Patients without underlying cirrhosis and those with stage $1 \mathrm{HCC}$ in the absence of thrombocytopenia $(<150,000 / \mathrm{cmm})$ and/or clinically significant portal hypertension are better served by resection. ${ }^{106,107}$ MELD score is a powerful predictor of outcomes following liver resection in cirrhotics. ${ }^{108}$ Post-hepatectomy liver failure (prothrombin time $<50 \%$ and serum bilirubin $>50 \mathrm{mmol} / \mathrm{dl}$ ) on day 5 after surgery was strong predictors of perioperative mortality. ${ }^{109}$ In one study, incidence of hepatic failure after hepatic resection was $0 \%$ with MELD score of $<9,3.6 \%$ with MELD 9-10, and $37.5 \%$ with MELD score of $>10$. In another study, perioperative mortality for minor (3 or less segments) or major (4 or more segments) hepatectomy was $29 \%$ amongst patients with MELD score of $>8$ and $0 \%$ with MELD score of 8 or less. Similarly, mortality after liver resection was higher for MELD score $>8$ compared to lower MELD ( $4 \%$ vs. $0.6 \% ; P=0.004$ ) on analyzing over 1100 HCC patients undergoing resection between 1991 and 2005 at one center in Taiwan. ${ }^{110}$ In yet another study, perioperative mortality was $19 \%$ at MELD > 8 vs. $0 \%$ for lower MELD. ${ }^{111}$ Same workers from Italy in another study reported on the concept of conditional survival (sum of the survival from the time of diagnosis and the postsurgical survival) and concluded that a MELD score of $<9$ achieves best conditional survival. Amongst patients with MELD >9, further risk stratification can be made based on extent of surgery and serum sodium levels. ${ }^{112}$

\section{Variceal Bleeding}

Many variables such as severity of liver disease, severity of bleeding, bleeding in the hospital, receipt of endoscopic treatment, hepatic venous pressure gradient (HVPG), and HCC predict outcome after variceal hemorrhage 
(VH). ${ }^{113-115}$ MELD score has been shown to be an accurate predictor of outcome after $\mathrm{VH} .{ }^{83,116,117}$ MELD >18 was a predictor of rebleeding within first 5 days and overall mortality at 6 weeks. ${ }^{117}$ MELD score is also a powerful predictor for mortality at 6 weeks for patients who develop early rebleeding after endoscopic variceal ligation. ${ }^{118} \mathrm{Com}$ parison of CTP and MELD scores in predicting outcome of $\mathrm{VH}$ have shown discrepant data with similar prediction in one study ${ }^{119}$ while CTP to be better predictor compared to MELD in another study. ${ }^{120}$

\section{Hepatorenal Syndrome}

Outcome depends on type of HRS with extremely poor prognosis for type 1 patients and MELD score of 20 or more. In contrast, patients with type $2 \mathrm{HRS}$ have longer survival if their MELD score is <20 compared to $\geq 20$ (11 vs. 3 months; $P<0.002)^{78}$

\section{Trauma}

MELD score is a predictor of death in trauma patients with each unit increase in MELD increasing mortality risk by $18 \%{ }^{121}$

\section{Re-transplantation}

Since implementation of the MELD score, the number of re-transplants has decreased raising the question as to whether MELD disadvantages patients listed for retransplantation. This issue was addressed in a study from the Mayo Clinic which showed that current MELD allocation policy serves candidates listed for primary or re-transplantation equitably. ${ }^{122}$

\section{IS MODEL FOR END-STAGE LIVER DISEASE AN IDEAL PROGNOSTIC SCORE?}

An ideal prognostic score should be accurate, objective, valid on a continuous scale, validated worldwide, should be able to guide treatment, and easy to calculate. MELD score meets most of these criteria with its biggest strength being validation across the world in various liver diseases. However, there are some limitations of the MELD score. Although, the variables needed to calculate the score are readily available, there is need for a computing device or website for calculating the score. The score has also potential for inaccuracy to predict waitlist mortality in $15-20 \%$ cases; therefore, further refinements are required before it can be considered an ideal score.

\section{FURTHER REFINEMENTS OF MODEL FOR END-STAGE LIVER DISEASE}

MELD variables can fluctuate with correction of precipitating factor such as control of infection, discontinuation of hepatotoxic drug or diuretics, and correction of renal dysfunction, coagulopathy, or biliary obstruction. When the MELD score was initially developed, the bilirubin, creatinine, and INR were recorded when the acute process has resolved. This issue was later addressed in a study where the serial MELD scores were taken until the MELD stabilized. The data showed that delta-MELD (difference between current MELD score and lowest MELD score within 30 days prior to current MELD) was not significant after controlling for other factors in the model. ${ }^{123}$ The authors concluded that it is reasonable to take current MELD for predicting survival in daily practice even in recently decompensated patients. However, in another study, deltaMELD was predictive of waitlist as well as post-LT survival with 4.9 odds of dying in the post-transplant period with delta-MELD of $>10 .{ }^{40}$

\section{Model for End-stage Liver Disease-Na}

Hyponatremia is a predictor of waitlist mortality amongst patients with end-stage liver disease after controlling for MELD score with increase in mortality by $5 \%$ for each mmol decrease in serum sodium between levels of 125 $140 \mathrm{mmol} / \mathrm{L}^{124}$ Serum sodium is a reflection of renal function and hypothetically may improve the accuracy of the model as serum creatinine used for calculating the MELD score may not accurately capture the true renal status. Similar observations about the impact of serum sodium between 125 and $140 \mathrm{mmol} / \mathrm{L}$ on the waitlist mortality have been made by other workers. ${ }^{125}$ Amongst patients with severe hyponatremia ( $<125 \mathrm{mmol} / \mathrm{L})$, serum sodium was a better predictor of mortality than MELD score amongst patients with refractory ascites. ${ }^{126}$

Data on the impact of sodium on the accuracy of MELD score are controversial as reported by other studies. ${ }^{127}$ In a recently reported study, addition of serum sodium into the MELD model very marginally improved the accuracy of the model with increase of c-statistic from 0.865 to $0.878(P<0.01)$ and in the validation data-set, MELD-Na affected only about $12 \%$ of listed patients. ${ }^{128}$ In another study reported from the Mayo Clinic, MELD-Na was better predictor of outcome amongst patients with alcoholic hepatitis who had ascites, but not in those without ascites. ${ }^{129}$ Lack of significant impact of serum sodium on the MELD score's accuracy could be due to the fact that a small proportion of patients with cirrhosis develop significant hyponatremia. For now, it is unclear whether liver organs should be allocated based on the MELD-Na model. Incorporation of serum sodium is also limited by its potential for 'manipulating' the system with change in volume status with free water intake and use of diuretics.

\section{Other Suggested Modifications}

Updated MELD (assigning lower weight to creatinine and INR while higher weight to bilirubin based on SRTR analysis of 38,899 patients transplanted between 2001- 
2006), ${ }^{130}$ refit MELD (reassigning lower and upper limits of 0.8 and 3.0 for serum creatinine while 1 and 3 for INR respectively), ${ }^{128}$ integrated MELD including sodium and age, ${ }^{93}$ MESO (ratio of MELD to serum sodium), Meld$\mathrm{Na}$ (incorporating serum sodium for levels between 125 and $140 \mathrm{mmol} / \mathrm{L}),{ }^{124} \mathrm{UK}$ end stage liver disease score (UKELD) which is similar to MELD-Na and is used for listing patients for liver transplantation in the $\mathrm{UK},{ }^{131}$ and ReFit MELD-Na as for refit MELD but including serum sodium also ${ }^{128}$ are some of the refinements, which have been tried to improve the accuracy of the MELD score. In one study, comparing these models, UKELD and updated MELD were poor in predicting mortality compared to other 4 models. ${ }^{32}$

Other factors shown to improve the MELD accuracy are HVPG ${ }^{133}$ von Willebrand factor level at a cut-off level of $315 \%,{ }^{134}$ persistent CRP levels of $\geq 29 \mathrm{mg} / \mathrm{L},{ }^{135}$ prealbumin levels of $>69 \mathrm{mg} / \mathrm{L},{ }^{136}$ apoptosis marker CK-18. ${ }^{137}$ Refinements to improve the accuracy of MELD would continue and until we find a better score, livers could continue to be allocated using the current MELD based system.

\section{CONFLICTS OF INTEREST}

All authors have none to declare.

\section{REFERENCES}

1. Lucey MR, Brown KA, Everson GT, et al. Minimal criteria for placement of adults on the liver transplant waiting list: a report of a national conference organized by the American Society of Transplant Physicians and the American Association for the Study of Liver Diseases. Liver Transpl Surg. 1997;3:628-637.

2. Van Meter $\mathrm{CH}$. The organ allocation controversy: how did we arrive here? Ochsner J. 1999;1:6-11.

3. Kamath PS, Wiesner RH, Malinchoc M, et al. A model to predict survival in patients with end-stage liver disease. Hepatology. 2001;33:464-470.

4. Malinchoc M, Kamath PS, Gordon FD, Peine CJ, Rank J, ter Borg PC. A model to predict poor survival in patients undergoing transjugular intrahepatic portosystemic shunts. Hepatology. 2000;31:864-871.

5. Brandsaeter B, Friman S, Broome U, et al. Outcome following liver transplantation for primary sclerosing cholangitis in the Nordic countries. Scand J Gastroenterol. 2003;38:1176-1183.

6. Said A, Williams J, Holden J, et al. Model for end stage liver disease score predicts mortality across a broad spectrum of liver disease. J Hepatol. 2004;40:897-903.

7. Wiesner R, Edwards E, Freeman R, et al. Model for end-stage liver disease (MELD) and allocation of donor livers. Gastroenterology. 2003;124:91-96.

8. Wiesner RH, McDiarmid SV, Kamath PS, et al. MELD and PELD: application of survival models to liver allocation. Liver Transpl. 2001;7:567-580.

9. Cholongitas E, Papatheodoridis GV, Vangeli M, Terreni N, Patch D, Burroughs AK. Systematic review: the model for end-stage liver disease - should it replace Child-Pugh's classification for assessing prognosis in cirrhosis? Aliment Pharmacol Ther. 2005;22:1079-1089.
10. Cholongitas E, Senzolo M, Patch D, et al. Risk factors, sequential organ failure assessment and model for end-stage liver disease scores for predicting short term mortality in cirrhotic patients admitted to intensive care unit. Aliment Pharmacol Ther. 2006;23:883-893.

11. Charlton MR, Wall WJ, Ojo AO, et al. Report of the first international liver transplantation society expert panel consensus conference on renal insufficiency in liver transplantation. Liver Transpl. 2009;15:S1-S34.

12. Sharma P, Welch K, Eikstadt R, Marrero JA, Fontana RJ, Lok AS. Renal outcomes after liver transplantation in the model for end-stage liver disease era. Liver Transpl. 2009;15: 1142-1148.

13. Nair S, Verma S, Thuluvath PJ. Pretransplant renal function predicts survival in patients undergoing orthotopic liver transplantation. Hepatology. 2002;35:1179-1185.

14. Gish RG. Do we need to MEND the MELD? Liver Transpl. 2007;13:486-487.

15. Asrani SK, Kim WR. Model for end-stage liver disease: end of the first decade. Clin Liver Dis. 2011;15:685-698.

16. Huo TI, Hsu CY, Lin HC, et al. Selecting an optimal cutoff value for creatinine in the model for end-stage liver disease equation. Clin Transplant. 2010;24:157-163.

17. Cholongitas E, Marelli L, Kerry A, et al. Different methods of creatinine measurement significantly affect MELD scores. Liver Transpl. 2007;13:523-529.

18. Cirillo M, Anastasio P, De Santo NG. Relationship of gender, age, and body mass index to errors in predicted kidney function. Nephrol Dial Transplant. 2005;20:1791-1798.

19. Leithead JA, MacKenzie SM, Ferguson JW, Hayes PC. Is estimated glomerular filtration rate superior to serum creatinine in predicting mortality on the waiting list for liver transplantation? Transplant Int. 2011;24:482-488.

20. Huo SC, Huo TI, Lin HC, et al. Is the corrected-creatinine model for end-stage liver disease a feasible strategy to adjust gender difference in organ allocation for liver transplantation? Transplantation. 2007;84:1406-1412.

21. Francoz C, Prie D, Abdelrazek W, et al. Inaccuracies of creatinine and creatinine-based equations in candidates for liver transplantation with low creatinine: impact on the model for end-stage liver disease score. Liver Transpl. 2010;16:1169-1177.

22. Schouten JN, Francque S, Van Vlierberghe H, et al. The influence of laboratory-induced MELD score differences on liver allocation: more reality than myth. Clin Transplant. 2012;26:E62-E70.

23. Trotter JF, Olson J, Lefkowitz J, Smith AD, Arjal R, Kenison J. Changes in international normalized ratio (INR) and model for endstage liver disease (MELD) based on selection of clinical laboratory. Am J Transplant. 2007;7:1624-1628.

24. Tripodi A, Chantarangkul V, Primignani M, et al. The international normalized ratio calibrated for cirrhosis (INR (liver)) normalizes prothrombin time results for model for end-stage liver disease calculation. Hepatology. 2007;46:520-527.

25. Heuman DM, Mihas AA, Habib A, et al. MELD-XI: a rational approach to "sickest first" liver transplantation in cirrhotic patients requiring anticoagulant therapy. Liver Transpl. 2007;13:30-37.

26. Botta F, Giannini E, Romagnoli P, et al. MELD scoring system is useful for predicting prognosis in patients with liver cirrhosis and is correlated with residual liver function: a European study. Gut. 2003;52:134-139.

27. Wiesner R, Lake JR, Freeman RB, Gish RG. Model for end-stage liver disease (MELD) exception guidelines. Liver Transpl. 2006;12:S85-S87.

28. Brown Jr RS, Lake JR. The survival impact of liver transplantation in the MELD era, and the future for organ allocation and distribution. Am J Transplant. 2005;5:203-204. 
29. Oberkofler CE, Dutkowski P, Stocker R, et al. Model of end stage liver disease (MELD) score greater than 23 predicts length of stay in the ICU but not mortality in liver transplant recipients. Crit Care. 2010;14:R117.

30. Dutkowski P, Oberkofler CE, Bechir M, et al. The model for endstage liver disease allocation system for liver transplantation saves lives, but increases morbidity and cost: a prospective outcome analysis. Liver Transpl. 2011;17:674-684.

31. Bambha K, Kim WR, Benson J, et al. Economic impact of meld-based allocation of liver transplants. Am J Transplant. 2005;5:425.

32. Aberg F, Maklin S, Rasanen P, et al. Cost of a quality-adjusted life year in liver transplantation: the influence of the indication and the model for end-stage liver disease score. Liver Transpl. 2011;17: 1333-1343.

33. Moylan CA, Brady CW, Johnson JL, Smith AD, Tuttle-Newhall JE, Muir AJ. Disparities in liver transplantation before and after introduction of the MELD score. JAMA. 2008;300:2371-2378.

34. Mathur AK, Schaubel DE, Gong Q, Guidinger MK, Merion RM. Racial and ethnic disparities in access to liver transplantation. Liver Transpl. 2010;16:1033-1040.

35. Myers RP, Shaheen AA, Aspinall Al, Quinn RR, Burak KW. Gender, renal function, and outcomes on the liver transplant waiting list: assessment of revised MELD including estimated glomerular filtration rate. J Hepatol. 2011;54:462-470.

36. Zhang M, Yin F, Chen B, et al. Pretransplant prediction of posttransplant survival for liver recipients with benign end-stage liver diseases: a nonlinear model. PloS ONE. 2012;7:e31256.

37. Rana A, Hardy MA, Halazun KJ, et al. Survival outcomes following liver transplantation (SOFT) score: a novel method to predict patient survival following liver transplantation. Am J Transplant. 2008;8:2537-2546.

38. Dutkowski P, Oberkofler CE, Slankamenac K, et al. Are there better guidelines for allocation in liver transplantation? A novel score targeting justice and utility in the model for end-stage liver disease era. Ann Surg. 2011;254:745-753. discussion 53.

39. Briceno J, Ciria R, de la Mata M, Rufian S, Lopez-Cillero P. Prediction of graft dysfunction based on extended criteria donors in the model for end-stage liver disease score era. Transplantation. 2010;90:530-539.

40. Gyori GP, Silberhumer GR, Zehetmayer S, et al. Dynamic changes in MELD score not only predict survival on the waiting list but also overall survival after liver transplantation. Transplant Int. 2012; 25:935-940.

41. Silberhumer GR, Pokorny $\mathrm{H}$, Hetz $\mathrm{H}$, et al. Combination of extended donor criteria and changes in the model for end-stage liver disease score predict patient survival and primary dysfunction in liver transplantation: a retrospective analysis. Transplantation. 2007;83:588-592.

42. Ling $Q, X u X$, Wei $Q$, et al. Downgrading MELD improves the outcomes after liver transplantation in patients with acute-onchronic hepatitis B liver failure. PIOS ONE. 2012;7:e30322.

43. Halldorson JB, Bakthavatsalam R, Fix O, Reyes JD, Perkins JD. D-MELD, a simple predictor of post liver transplant mortality for optimization of donor/recipient matching. Am J Transplant. 2009;9:318-326.

44. Weismuller TJ, Fikatas P, Schmidt J, et al. Multicentric evaluation of model for end-stage liver disease-based allocation and survival after liver transplantation in Germany - limitations of the 'sickest first'-concept. Transplant Int. 2011;24:91-99.

45. Angermayr B, Luca A, Konig F, et al. Aetiology of cirrhosis of the liver has an impact on survival predicted by the model of endstage liver disease score. Eur J Clin Invest. 2009;39:65-71.

46. Ahmad J, Bryce CL, Cacciarelli T, Roberts MS. Differences in access to liver transplantation: disease severity, waiting time, and transplantation center volume. Ann Intern Med. 2007;146: 707-713.

47. Argo CK, Stukenborg GJ, Schmitt TM, Kumer SC, Berg CL, Northup PG. Regional variability in symptom-based MELD exceptions: a response to organ shortage? Am J Transplant. 2011; 11:2353-2361.

48. Ravaioli M, Masetti M, Ridolfi L, et al. Laboratory test variability and model for end-stage liver disease score calculation: effect on liver allocation and proposal for adjustment. Transplantation. 2007:83:919-924.

49. Ioannou GN, Perkins JD, Carithers Jr RL. Liver transplantation for hepatocellular carcinoma: impact of the MELD allocation system and predictors of survival. Gastroenterology. 2008;134: 1342-1351.

50. Wiesner RH, Freeman RB, Mulligan DC. Liver transplantation for hepatocellular cancer: the impact of the MELD allocation policy. Gastroenterology. 2004;127:S261-S267.

51. Washburn K, Edwards E, Harper A, Freeman R. Hepatocellular carcinoma patients are advantaged in the current liver transplant allocation system. Am J Transplant. 2010;10:1643-1648.

52. Massie AB, Caffo B, Gentry SE, et al. MELD exceptions and rates of waiting list outcomes. Am J Transplant. 2011;11:2362-2371.

53. Park CW, Tsai NT, Wong LL. Implications of worse renal dysfunction and medical comorbidities in patients with NASH undergoing liver transplant evaluation: impact on MELD and more. Clin Transplant. 2011;25:E606-E611.

54. Cucchetti A, Cescon M, Bertuzzo V, et al. Can the dropout risk of candidates with hepatocellular carcinoma predict survival after liver transplantation? Am J Transplant. 2011;11:1696-1704.

55. Toso C, Dupuis-Lozeron E, Majno P, et al. A model for dropout assessment of candidates with or without hepatocellular carcinoma on a common liver transplant waiting list. Hepatology. 2012;56: 149-156.

56. Nadim MK, Sung RS, Davis CL, et al. Simultaneous liver-kidney transplantation Summit: current state and future directions. Am J Transplant; 2012.

57. Singal A, Wiesner RH. Contemporary predictors of simultaneous liver kidney transplantation and impact on liver graft and patient outcomes. Gastroenterology. 2012;142:S433.

58. Sharma P, Schaubel DE, Guidinger MK, Goodrich NP, Ojo AO, Merion RM. Impact of MELD-based allocation on end-stage renal disease after liver transplantation. Am J Transplant. 2011;11: 2372-2378.

59. Eason JD, Gonwa TA, Davis CL, Sung RS, Gerber D, Bloom RD. Proceedings of consensus conference on simultaneous liver kidney transplantation (SLK). Am J Transplant. 2008;8:2243-2251.

60. Merion RM, Schaubel DE, Dykstra DM, Freeman RB, Port FK, Wolfe RA. The survival benefit of liver transplantation. Am J Transplant. 2005;5:307-313.

61. Wiencke K, Boberg KM. Current consensus on the management of primary sclerosing cholangitis. Clin Res Hepatol Gastroenterol. 2011;35:786-791.

62. Volk ML, Lok AS, Pelletier SJ, Ubel PA, Hayward RA. Impact of the model for end-stage liver disease allocation policy on the use of high-risk organs for liver transplantation. Gastroenterology. 2008;135:1568-1574.

63. Axelrod DA, Lentine KL, Salvalaggio PR, Schnitzler MA. The cost and quality paradox. Am J Transplant. 2009;9:985-986.

64. Antaki F, Lukowski A. The model for end-stage liver disease (MELD) predicts survival of liver cirrhosis patients after discharge to hospice. J Clin Gastroenterol. 2007;41:412-415.

65. Medici V, Rossaro L, Wegelin JA, et al. The utility of the model for end-stage liver disease score: a reliable guide for liver transplant candidacy and, for select patients, simultaneous hospice referral. Liver Transpl. 2008;14:1100-1106. 
66. Kremers WK, van IM, Kim WR, et al. MELD score as a predictor of pretransplant and posttransplant survival in OPTN/UNOS status 1 patients. Hepatology. 2004;39:764-769.

67. Katoonizadeh A, Decaestecker J, Wilmer A, et al. MELD score to predict outcome in adult patients with non-acetaminopheninduced acute liver failure. Liver Int. 2007;27:329-334.

68. Taylor RM, Davern T, Munoz S, et al. Fulminant hepatitis A virus infection in the United States: incidence, prognosis, and outcomes. Hepatology. 2006;44:1589-1597.

69. Schmidt LE, Larsen FS. MELD score as a predictor of liver failure and death in patients with acetaminophen-induced liver injury. Hepatology. 2007;45:789-796.

70. Sharma P, Schaubel DE, Gong Q, Guidinger M, Merion RM. End-stage liver disease candidates at the highest model for end-stage liver disease scores have higher wait-list mortality than status-1A candidates. Hepatology. 2012;55:192-198.

71. Sheth M, Riggs M, Patel T. Utility of the Mayo end-stage liver disease (MELD) score in assessing prognosis of patients with alcoholic hepatitis. BMC Gastroenterol. 2002;2:2.

72. Srikureja W, Kyulo NL, Runyon BA, Hu KQ. MELD score is a better prognostic model than Child-Turcotte-Pugh score or discriminant function score in patients with alcoholic hepatitis. J Hepatol. 2005;42:700-706.

73. Zapata-Irrison L, Jurado-Nunez J, Altamirano-Gomez J. [do MELD or Maddrey?: comparison of 2 forecasting models in patients with hepatitis toxic alcohol.]. Revista de gastroenterologia de Mexico. 2008;73:57-62.

74. Dunn W, Jamil LH, Brown LS, et al. MELD accurately predicts mortality in patients with alcoholic hepatitis. Hepatology. 2005;41: 353-358.

75. Verma S, Ajudia K, Mendler M, Redeker A. Prevalence of septic events, type 1 hepatorenal syndrome, and mortality in severe alcoholic hepatitis and utility of discriminant function and MELD score in predicting these adverse events. Dig Dis Sci. 2006;51: 1637-1643.

76. Jeong JY, Sohn JH, Son BK, et al. [Comparison of model for end-stage liver disease score with discriminant function and Child-Turcotte-Pugh scores for predicting short-term mortality in Korean patients with alcoholic hepatitis]. Korean J Gastroenterol = Taehan Sohwagi Hakhoe Chi. 2007;49:93-99.

77. O'Shea RS, Dasarathy S, McCullough AJ. Alcoholic liver disease. Hepatology. 2010;51:307-328.

78. Alessandria C, Ozdogan 0 , Guevara M, et al. MELD score and clinical type predict prognosis in hepatorenal syndrome: relevance to liver transplantation. Hepatology. 2005;41:1282-1289.

79. Kraja B, Sina M, Mone I, et al. Predictive value of the model of end-stage liver disease in cirrhotic patients with and without spontaneous bacterial peritonitis. Gastroenterol Res Pract. 2012; 2012:539059.

80. Obstein KL, Campbell MS, Reddy KR, Yang YX. Association between model for end-stage liver disease and spontaneous bacterial peritonitis. Am J Gastroenterol. 2007;102: 2732-2736.

81. Viasus D, Garcia-Vidal C, Castellote J, et al. Community-acquired pneumonia in patients with liver cirrhosis: clinical features, outcomes, and usefulness of severity scores. Medicine. 2011;90: 110-118.

82. Chen $\mathrm{CH}$, Shih $\mathrm{CM}$, Chou JW, et al. Outcome predictors of cirrhotic patients with spontaneous bacterial empyema. Liver Int. 2011; 31:417-424.

83. Chalasani N, Kahi C, Francois F, et al. Model for end-stage liver disease (MELD) for predicting mortality in patients with acute variceal bleeding. Hepatology. 2002;35:1282-1284.

84. Schepke M, Appenrodt B, Heller J, Zielinski J, Sauerbruch T. Prognostic factors for patients with cirrhosis and kidney dysfunction in the era of MELD: results of a prospective study. Liver Int. 2006;26:834-839.

85. Singal A, Kamath PS. Variceal Hemorrhage. New Delhi, India: Elsevier; 2012.

86. Feyssa E, Ortiz J, Grewal K, et al. MELD score less than 15 predicts prolonged survival after transjugular intrahepatic portosystemic shunt for refractory ascites after liver transplantation. Transplantation. 2011;91:786-792.

87. Garrison RN, Cryer HM, Howard DA, Polk Jr HC. Clarification of risk factors for abdominal operations in patients with hepatic cirrhosis. Ann Surg. 1984;199:648-655.

88. O'Leary JG, Yachimski PS, Friedman LS. Surgery in the patient with liver disease. Clin Liver Dis. 2009;13:211-231.

89. Prediction of the first variceal hemorrhage in patients with cirrhosis of the liver and esophageal varices. A prospective multicenter study. N Engl J Med. 1988;319:983-989.

90. Mansour A, Watson W, Shayani V, Pickleman J. Abdominal operations in patients with cirrhosis: still a major surgical challenge. Surgery. 1997;122:730-735. discussion 5-6.

91. Causey MW, Steele SR, Farris Z, Lyle DS, Beitler AL. An assessment of different scoring systems in cirrhotic patients undergoing nontransplant surgery. Am J Surg. 2012;203: 589-593.

92. Teh SH, Nagorney DM, Stevens SR, et al. Risk factors for mortality after surgery in patients with cirrhosis. Gastroenterology. 2007; 132:1261-1269.

93. Kim SH, Han YD, Lee JG, et al. MELD-based indices as predictors of mortality in chronic liver disease patients who undergo emergency surgery with general anesthesia. J Gastrointest Surg. 2011;15:2029-2035.

94. Costa BP, Sousa FC, Serodio M, Carvalho C. Value of MELD and MELD-based indices in surgical risk evaluation of cirrhotic patients: retrospective analysis of 190 cases. World J Surg. 2009;33:1711-1719.

95. Kim SY, Yim HJ, Park SM, et al. Validation of a Mayo post-operative mortality risk prediction model in Korean cirrhotic patients. Liver Int. 2011;31:222-228.

96. Cho HC, Jung HY, Sinn DH, et al. Mortality after surgery in patients with liver cirrhosis: comparison of Child-Turcotte-Pugh, MELD and MELD Na score. Eur J Gastroenterol Hepatol. 2011;23: 51-59.

97. Neeff H, Mariaskin D, Spangenberg HC, Hopt UT, Makowiec F. Perioperative mortality after non-hepatic general surgery in patients with liver cirrhosis: an analysis of 138 operations in the 2000s using Child and MELD scores. J Gastrointest Surg. 2011;15:1-11.

98. Hoteit MA, Ghazale AH, Bain AJ, et al. Model for end-stage liver disease score versus Child score in predicting the outcome of surgical procedures in patients with cirrhosis. World J Gastroenterol. 2008;14:1774-1780.

99. Delis S, Bakoyiannis A, Madariaga J, Bramis J, Tassopoulos N, Dervenis C. Laparoscopic cholecystectomy in cirrhotic patients: the value of MELD score and Child-Pugh classification in predicting outcome. Surg Endosc. 2010;24:407-412.

100. Kao HK, Guo LF, Cheng MH, et al. Predicting postoperative morbidity and mortality by model for endstage liver disease score for patients with head and neck cancer and liver cirrhosis. Head Neck. 2011;33:529-534.

101. Filsoufi F, Salzberg SP, Rahmanian PB, et al. Early and late outcome of cardiac surgery in patients with liver cirrhosis. Liver Transpl. 2007;13:990-995.

102. Marrocco-Trischitta MM, Kahlberg A, Astore D, Tshiombo G, Mascia D, Chiesa R. Outcome in cirrhotic patients after elective surgical repair of infrarenal aortic aneurysm. I Vasc Surg. 2011;53:906-911. 
103. Ailawadi G, Lapar DJ, Swenson BR, et al. Model for end-stage liver disease predicts mortality for tricuspid valve surgery. Ann Thorac Surg. 2009;87:1460-1467. discussion 7-8.

104. Bonde P, Ku NC, Genovese EA, et al. Model for end-stage liver disease score predicts adverse events related to ventricular assist device therapy. Ann Thorac Surg. 2012;93:1541-1547. discussion 7-8.

105. Matthews JC, Pagani FD, Haft JW, Koelling TM, Naftel DC, Aaronson KD. Model for end-stage liver disease score predicts left ventricular assist device operative transfusion requirements, morbidity, and mortality. Circulation. 2010;121:214-220.

106. Maithel SK, Kneuertz PJ, Kooby DA, et al. Importance of low preoperative platelet count in selecting patients for resection of hepatocellular carcinoma: a multi-institutional analysis. J Am Coll Surg. 2011;212:638-648. discussion 48-50.

107. Bruix J, Sherman M. Management of hepatocellular carcinoma: an update. Hepatology. 2011;53:1020-1022.

108. Teh SH, Sheppard BC, Schwartz J, Orloff SL. Model for end-stage liver disease score fails to predict perioperative outcome after hepatic resection for hepatocellular carcinoma in patients without cirrhosis. Am J Surg. 2008;195:697-701.

109. Rahbari NN, Garden OJ, Padbury R, et al. Posthepatectomy liver failure: a definition and grading by the International Study Group of Liver Surgery (ISGLS). Surgery. 2011;149:713-724.

110. Hsu KY, Chau GY, Lui WY, Tsay SH, King KL, Wu CW. Predicting morbidity and mortality after hepatic resection in patients with hepatocellular carcinoma: the role of model for end-stage liver disease score. World J Surg. 2009;33:2412-2419.

111. Delis SG, Bakoyiannis A, Biliatis I, Athanassiou K, Tassopoulos N, Dervenis C. Model for end-stage liver disease (MELD) score, as a prognostic factor for post-operative morbidity and mortality in cirrhotic patients, undergoing hepatectomy for hepatocellular carcinoma. HPB. 2009;11:351-357.

112. Cescon M, Cucchetti A, Grazi GL, et al. Indication of the extent of hepatectomy for hepatocellular carcinoma on cirrhosis by a simple algorithm based on preoperative variables. Arch Surg. 2009;144: 57-63. discussion.

113. Garcia-Tsao G, Bosch J. Management of varices and variceal hemorrhage in cirrhosis. N Engl J Med. 2010;362:823-832.

114. Singal AK, Jampana S, Singal V, Kuo YF. Presence of hepatocellular carcinoma is a predictor of in-patient mortality from acute variceal hemorrhage amongst patients with cirrhosis: analysis of National Inpatient Sample (1998-2007). Hepatology. 2011;54: 1368A-1369A.

115. Cerqueira RM, Andrade L, Correia MR, Fernandes CD, Manso MC. Risk factors for in-hospital mortality in cirrhotic patients with oesophageal variceal bleeding. Eur J Gastroenterol Hepatol. 2012;24: 551-557.

116. Amitrano L, Guardascione MA, Bennato R, Manguso F, Balzano A. MELD score and hepatocellular carcinoma identify patients at different risk of short-term mortality among cirrhotics bleeding from esophageal varices. J Hepatol. 2005;42:820-825.

117. Bambha K, Kim WR, Pedersen R, Bida JP, Kremers WK, Kamath PS. Predictors of early re-bleeding and mortality after acute variceal haemorrhage in patients with cirrhosis. Gut. 2008;57:814-820.

118. Chen WT, Lin CY, Sheen IS, et al. MELD score can predict early mortality in patients with rebleeding after band ligation for variceal bleeding. World J Gastroenterol. 2011;17:2120-2125.

119. Flores-Rendon AR, Gonzalez-Gonzalez JA, Garcia-Compean D, Maldonado-Garza HJ, Garza-Galindo AA. Model for end stage of liver disease (MELD) is better than the Child-Pugh score for pre- dicting in-hospital mortality related to esophageal variceal bleeding. Ann Hepatol. 2008;7:230-234.

120. Orloff MJ, Vaida F, Isenberg Jl, et al. Child-Turcotte score versus MELD for prognosis in a randomized controlled trial of emergency treatment of bleeding esophageal varices in cirrhosis. J Surg Res; 2012.

121. Inaba K, Barmparas G, Resnick S, et al. The model for end-stage liver disease score: an independent prognostic factor of mortality in injured cirrhotic patients. Arch Surg. 2011;146:1074-1078.

122. Kim HJ, Larson JJ, Lim YS, et al. Impact of MELD on waitlist outcome of retransplant candidates. Am J Transplant. 2010;10: 2652-2657.

123. Bambha K, Kim WR, Kremers WK, et al. Predicting survival among patients listed for liver transplantation: an assessment of serial MELD measurements. Am J Transplant. 2004;4:1798-1804.

124. Kim WR, Biggins SW, Kremers WK, et al. Hyponatremia and mortality among patients on the liver-transplant waiting list. $N$ Engl J Med. 2008;359:1018-1026.

125. Moini M, Hoseini-AsI MK, Taghavi SA, et al. Hyponatremia a valuable predictor of early mortality in patients with cirrhosis listed for liver transplantation. Clin Transplant. 2011;25:638-645.

126. Serste T, Gustot T, Rautou PE, et al. Severe hyponatremia is a better predictor of mortality than MELD Na in patients with cirrhosis and refractory ascites. J Hepatol. 2012;57:274-280.

127. Londono MC, Cardenas A, Guevara M, et al. MELD score and serum sodium in the prediction of survival of patients with cirrhosis awaiting liver transplantation. Gut. 2007;56:1283-1290.

128. Leise MD, Kim WR, Kremers WK, Larson JJ, Benson JT, Therneau TM. A revised model for end-stage liver disease optimizes prediction of mortality among patients awaiting liver transplantation. Gastroenterology. 2011;140:1952-1960.

129. Vaa BE, Asrani SK, Dunn W, Kamath PS, Shah VH. Influence of serum sodium on MELD-based survival prediction in alcoholic hepatitis. Mayo Clin Proc. 2011;86:37-42.

130. Sharma P, Schaubel DE, Sima CS, Merion RM, Lok AS. Re-weighting the model for end-stage liver disease score components. Gastroenterology. 2008;135:1575-1581.

131. Barber K, Madden S, Allen J, Collett D, Neuberger J, Gimson A. Elective liver transplant list mortality: development of a United Kingdom end-stage liver disease score. Transplantation. 2011; 92:469-476.

132. Magder LS, Regev A, Mindikoglu AL. Comparison of seven liver allocation models with respect to lives saved among patients on the liver transplant waiting list. Transplant Int. 2012;25:409-415.

133. Suk KT, Kim CH, Park SH, et al. Comparison of hepatic venous pressure gradient and two models of end-stage liver disease for predicting the survival in patients with decompensated liver cirrhosis. J Clin Gastroenterol; 2012.

134. Ferlitsch M, Reiberger $\mathrm{T}$, Hoke $\mathrm{M}$, et al. Von Willebrand factor as new non-invasive predictor of portal hypertension, decompensation and mortality in patients with liver cirrhosis. Hepatology; 2012.

135. Cervoni JP, Thevenot T, Weil D, et al. C-reactive protein predicts short-term mortality in patients with cirrhosis. I Hepatol. 2012;56:1299-1304.

136. Liu F, Cai LY, Zhong L, et al. Model for end-stage liver disease combined with serum prealbumin to predict the prognosis of patients with decompensated liver cirrhosis. J Dig Dis. 2010;11:352-357.

137. Bechmann LP, Jochum C, Kocabayoglu P, et al. Cytokeratin 18-based modification of the MELD score improves prediction of spontaneous survival after acute liver injury. J Hepatol. 2010;53:639-647. 